\title{
Bound states in the continuum and Fano resonances in the strong mode coupling regime
}

\author{
Andrey A. Bogdanov, ${ }^{a, b}$ Kirill L. Koshelev, ${ }^{a, c}$ Polina V. Kapitanova, ${ }^{a}$ Mikhail V. Rybin, ${ }^{a, b}$ Sergey A. Gladyshev, ${ }^{a}$ \\ Zarina F. Sadrieva, ${ }^{a}$ Kirill B. Samusev ${ }^{\mathrm{a}, \mathrm{b}}$ Yuri S. Kivshar, ${ }^{\mathrm{a}, \mathrm{c}, \mathrm{t}}$ and Mikhail F. Limonov ${ }^{\mathrm{a}, \mathrm{b}}$ \\ ITMO University, Department of Nanophotonics and Metamaterials, St. Petersburg, Russia \\ bloffe Institute, St. Petersburg, Russia \\ ${ }^{c}$ Australian National University, Nonlinear Physics Center, Canberra, Australia
}

\begin{abstract}
The study of resonant dielectric nanostructures with a high refractive index is a new research direction in the nanoscale optics and metamaterial-inspired nanophotonics. Because of the unique optically induced electric and magnetic Mie resonances, high-index nanoscale structures are expected to complement or even replace different plasmonic components in a range of potential applications. We study a strong coupling between modes of a single subwavelength high-index dielectric resonator and analyze the mode transformation and Fano resonances when the resonator's aspect ratio varies. We demonstrate that strong mode coupling results in resonances with high-quality factors, which are related to the physics of bound states in the continuum when the radiative losses are almost suppressed due to the Friedrich-Wintgen scenario of destructive interference. We explain the physics of these states in terms of multipole decomposition, and show that their appearance is accompanied by a drastic change in the far-field radiation pattern. We reveal a fundamental link between the formation of the high-quality resonances and peculiarities of the Fano parameter in the scattering cross-section spectra. Our theoretical findings are confirmed by microwave experiments for the scattering of high-index cylindrical resonators with a tunable aspect ratio. The proposed mechanism of the strong mode coupling in single subwavelength high-index resonators accompanied by resonances with high-quality factors helps to extend substantially functionalities of all-dielectric nanophotonics, which opens horizons for active and passive nanoscale metadevices.
\end{abstract}

Keywords: Fano resonance; bound states in the continuum; Mie resonance; dielectric resonator; meta-optics.

Received Oct. 31, 2018; accepted for publication Dec. 3, 2018; published online Jan. 28, 2019.

(C) The Authors. Published by SPIE and CLP under a Creative Commons Attribution 4.0 Unported License. Distribution or reproduction of this work in whole or in part requires full attribution of the original publication, including its DOI.

[DOI: 10.1117/1.AP.1.1.016001]

\section{Introduction}

The physics of resonant structures with a strong mode coupling is of fundamental importance, and is responsible for a variety of interesting phenomena governing both transport and localization of waves. The modes supported by traditional resonators and microcavities ${ }^{1}$ exist due to the reflection of waves from the resonator's boundaries under the conditions of constructive interference. To achieve high values for the resonator's quality factor ( $Q$-factor), one can improve reflectivity, whether by using metals, ${ }^{2,3}$ photonic bandgap structures, ${ }^{4}$ or the total internal reflection at glancing angles of incidence in whispering-gallery-mode

*Address all correspondence to Yuri S. Kivshar, E-mail: ysk@internode.on.net
(WGM) resonators. ${ }^{5}$ Such physical mechanisms require large-sized cavities with a complex design. A more attractive way to confine light is to use destructive interference in the regime of strong mode coupling..$^{6-8}$ This mechanism is related to the physics of bound states in the continuum (BIC) ${ }^{9}$ It was first proposed in quantum mechanics by Friedrich and Wintgen ${ }^{10}$ and then was extended to acoustics ${ }^{11-13}$ and electrodynamics. ${ }^{14,15}$ A true optical BIC is a mathematical abstraction, as its realization demands either infinite size of the structure or zero (or infinite) permittivity. ${ }^{16-18}$ However, the BIC-inspired mechanism of light localization makes possible realization of high- $Q$ states in photonic crystal cavities and slabs, ${ }^{15,17,19}$ coupled waveguide arrays, ${ }^{20-22}$ dielectric gratings,${ }^{14}$ core-shell spherical particles,${ }^{18}$ dielectric resonators, ${ }^{23-26}$ and hybrid plasmonic-photonic systems. ${ }^{27}$ 
By definition, the $Q$-factor of a true BIC is infinite. Hence, in the wave scattering, BIC manifests itself as a "collapse" of the Fano resonance when the width of the resonance vanishes and the Fano feature disappears from the scattering spectrum. ${ }^{28,29}$ In practice, both $Q$-factor and the width of the Fano resonance at the frequency of BIC remain finite because of absorption, finitesize samples, roughness, and other imperfections. ${ }^{30}$ Remarkably, in terms of the scattering matrix, BIC corresponds to merging of a pole and a zero of the scattering operator on the real axis. ${ }^{31}$ The properties of the Fano resonances in the systems with BICs have been considered in several studies. . $^{15,17,18}$

A conventional device supporting light localization via a BIC-inspired mechanism is based on periodic photonic structures ${ }^{17}$ or chains of scatterers. ${ }^{32}$ For these structures, strong localization can be achieved only for a large number of scatterers because it is governed by their mutual interference. Other implementations of BICs in photonic structures were presented in Refs. 18 and 25. In the former work (Ref. 18), the design is based on a metallic box with a single dielectric scatterer inside. Although the conditions of true BICs can be achieved here by imitating periodic boundary conditions for such a unit cell, the structure is not subwavelength, and it demands using metallic components. The latter work (Ref. 25) describes a single scatterer, but it employs near-zero refraction index constituents that require large scales of the structure, ${ }^{33}$ especially, at optical frequencies. Recently, BIC-inspired supercavity modes in the subwavelength dielectric resonators without singular permittivity values were proposed theoretically. ${ }^{8}$ However, the coexistence of the Fano resonance as the effect of weak coupling and strong mode coupling underlying BIC is a vital problem of modern photonics.

In this paper, we demonstrate, both theoretically and experimentally, strong light localization and existence of quasi-BICs in the simplest object-a single homogeneous cylindrical subwavelength dielectric resonator in free space. We show that quasi-BICs appear in accord with the Friedrich-Wintgen interference mechanism because of strong coupling between Mielike and Fabry-Perot-like modes. We develop an analytical approach to describe light scattering by finite-size dielectric resonators and reveal close relationships between the peculiarities of quasi-BICs and the critical behavior of the Fano asymmetry parameter in the strong coupling regime. We show that the Fano
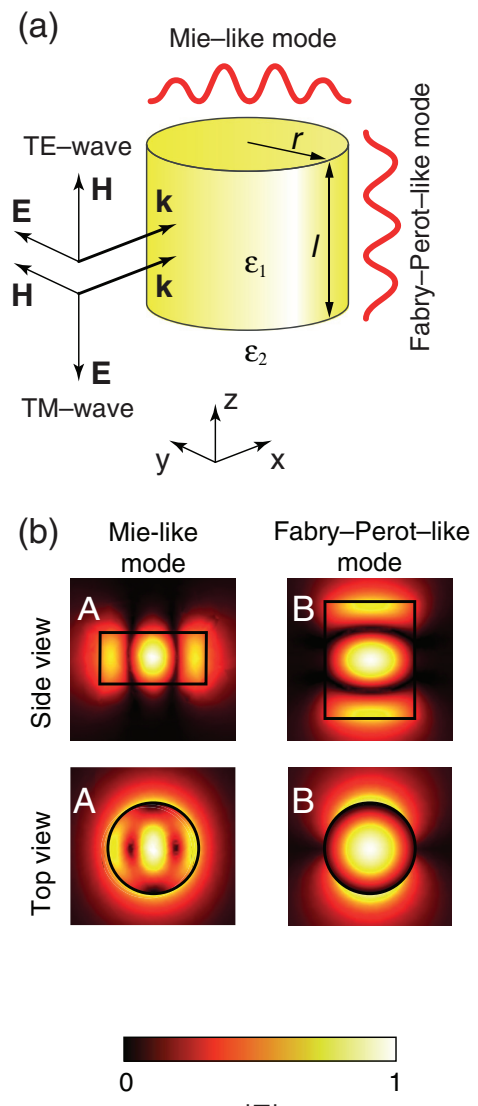

|E|
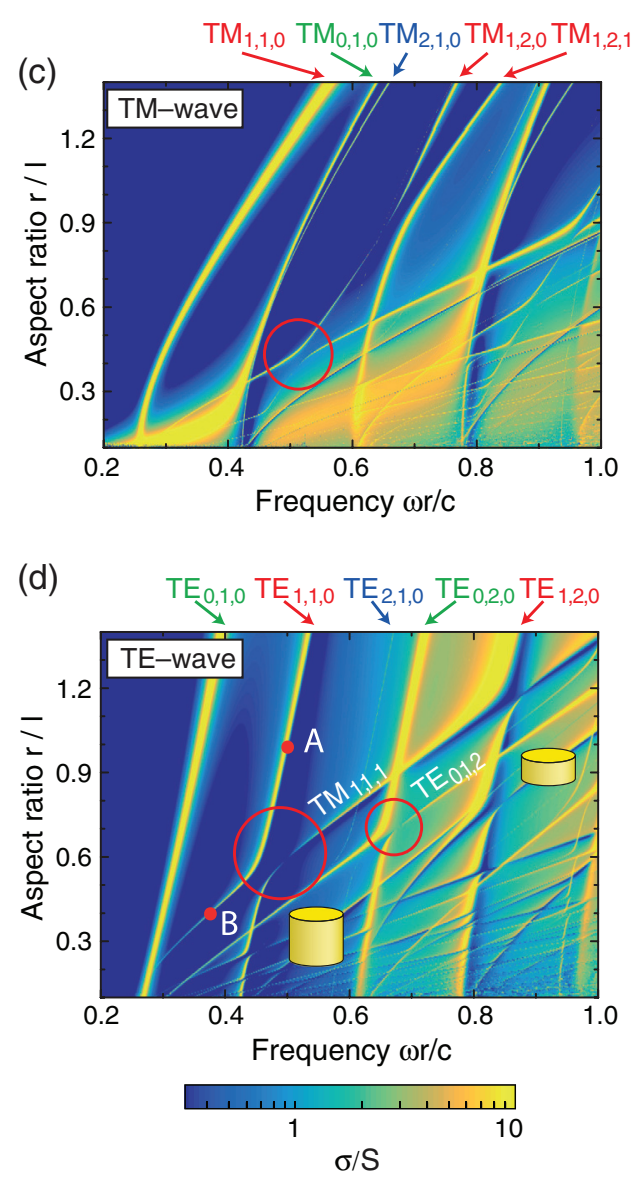

Fig. 1 Strong coupling of modes in a dielectric resonator. (a) TE- and TM-polarized waves incident on a dielectric cylindrical resonator with permittivity $\varepsilon_{1}=80$, radius $r$, and length / placed in vacuum $\left(\varepsilon_{2}=1\right)$. (b) Distribution of the electric field amplitude $|E|$ for the Mie-like mode $\mathrm{TE}_{1,1,0}$ (point A) and Fabry-Perot-like mode $\mathrm{TM}_{1,1,1}$ (point B). (c) and (d) Dependencies of the total SCS of the cylinder $\sigma$ normalized to the projected cross-section $S=2 r l$ on the aspect ratio of the cylinder and frequency $r \omega / c=2 \pi r / \lambda$ for TM and TE-polarized incident waves, respectively. The calculations are carried out with the step of $r / I=0.003$. In panels (c) and (d), the regions of the most pronounced avoided crossing are marked by red circles. 
asymmetry parameter becomes singular at the frequency of the quasi-BIC, and it vanishes when the mode becomes almost dark for far-field excitation. We derive an exact form of coupling coefficients between modes and corresponding Rabi frequencies. We analyze effects of material losses and reveal that the mode coupling remains strong even for highly absorptive resonators. Our findings make evident the counterintuitive fact that even a subwavelength dielectric resonator could provide strong light localization.

\section{Results}

\subsection{Interplay of Mie and Fabry-Perot Modes}

We start our study with numerical simulations of the scattering cross-section (SCS) $\sigma$ of a finite dielectric cylinder with permittivity $\varepsilon_{1}=80$, radius $r$, and length $l$ placed in vacuum $\left(\varepsilon_{2}=1\right)$, as shown in Fig. 1(a). The spectra are calculated by using the CST Microwave Studio software and T-matrix computations. ${ }^{34,35}$ The electric field of the incident wave is assumed to be perpendicular to the axis of the cylinder [see Fig. 1(a)]. To compare $\sigma$ for cylinders with different aspect ratios, we normalize $\sigma$ by the projected cross section of the resonator, $S=2 r l$. The maps of the normalized SCS $\sigma / S$ calculated for cylinders with different aspect ratios $r / l$ excited by TM and TE-polarized waves are shown in Figs. 1(c) and 1(d), respectively. According to the standard nomenclature (see, e.g., Ref. 36), we denote the modes of a cylindrical resonator as $\mathrm{TE}_{n, k, p}$ and $\mathrm{TM}_{n, k, p}$, where $n, k, p$ are the indices denoting the azimuthal, radial, and axial wavenumbers, respectively. Generally speaking, distinguishing between $\mathrm{TE}_{n, k, p}$ and $\mathrm{TM}_{n, k, p}$ modes for a cylinder of a finite length is justified only for $n=0$. For other cases, the polarization is hybrid. ${ }^{37}$ In the case of arbitrary $n, k, p$, the mode polarization is mixed. Thus, under the terms "TE" or "TM," we further imply the dominant polarization of the modes.

The low-frequency spectrum of the dielectric cylinder under consideration consists of three types of modes. The modes with the axial index $p=0$ and azimuthal index $n=0,1$ demonstrate a low-frequency shift with changing $r / l$. They are formed mainly due to reflection from a side wall of the cylinder, and they could be associated with the Mie resonances of an infinite cylinder [see Figs. 1(a) and 2(a)]. The modes with the indices $p>0$ and $n=0,1$ demonstrate a strong shift to higher frequencies with increasing aspect ratio of $r / l$. They are formed mainly due to reflection from the faces of the cylinder, and they could be associated with the Fabry-Perot modes [see Figs. 1(a) and 2(a)]. The modes with the azimuthal index $n=2,3, \ldots$ are formed due to the wave incident on the side wall of the cylinder at the angles bigger than the total internal reflection angle, which is about $6.4 \mathrm{deg}$ for $\varepsilon_{1}=80$. Therefore, they are close in nature to the whispering gallery modes [see Fig. 2(a)] and their high $Q$-factor is explained by total internal reflection but not by destructive interference as we have for quasi-BIC. The properties of WGMs are well studied (see, e.g., Refs. 5, 38, and 39); we focus on the Mie-like $\left(\mathrm{TE}_{1,1,0}\right)$ and FabryPerot-like $\left(\mathrm{TM}_{1,1,1}\right)$ modes. Their electric field distributions are shown in Fig. 1(b).

In quantum mechanics, in the simplest case, the system with light-matter interaction is described by a sum of Hamiltonian without interaction $\hat{H}_{0}$ and an interaction potential $\hat{V}$ (see, e.g., Ref. 40). The diagonal components of $\hat{V}$ are responsible for the energy shift, and the off-diagonal components are (a)
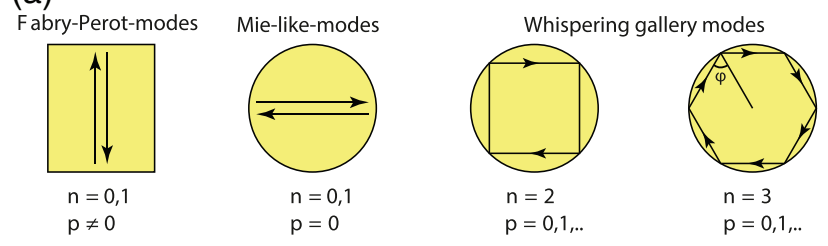

(b)
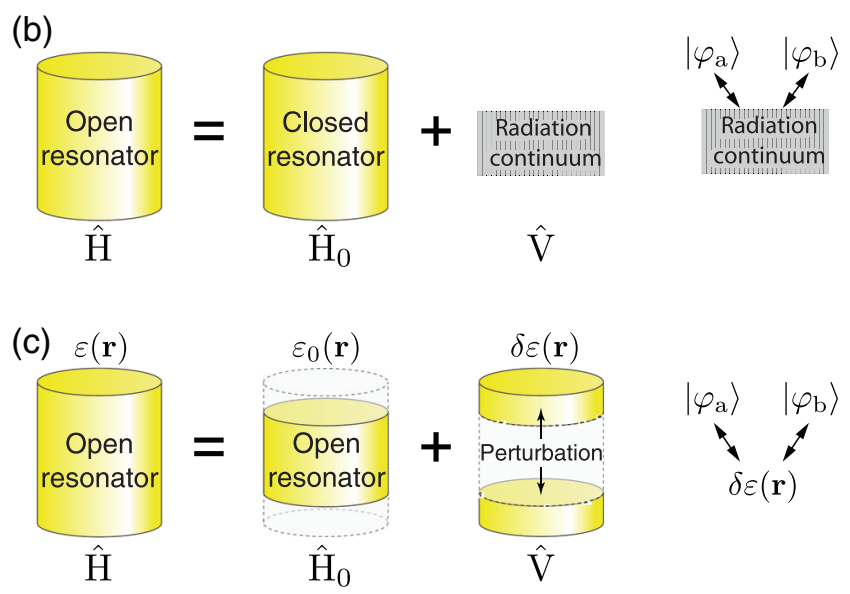

Fig. 2 Modes of a dielectric resonator and models of their coupling. (a) Classification of eigenmodes of a dielectric resonator. (b) Friedrich-Wintgen approach describing an open cylindrical resonator as a closed resonator and a radiation continuum. Eigenmodes of the resonator interact via the radiation continuum. (c) Non-Hermitian approach describing an open cylindrical resonator by a complex spectrum of eigenfrequencies. Eigenmodes of the resonator interact via perturbation $\delta \varepsilon(r)$ responsible for change in the resonator aspect ratio.

responsible for the coupling. The interaction results in a mixing of the light and matter states and in appearance of an avoided resonance crossing - the characteristic feature of the strong coupling regime. ${ }^{41}$

In electromagnetism, due to the fact that a resonator is an open system, description of the interaction between the modes becomes more complicated. There are two main approaches describing the interaction between the modes in an open system. The first one considers an open system (dielectric cylindrical resonator in our case) as a closed system with nonradiating modes $\left|\varphi_{a}\right\rangle$ and $\left|\varphi_{b}\right\rangle$ interacting with a continuum of the radiation modes outside of the resonator in accord with the Friedrich-Wintgen mechanism ${ }^{10}$ [Fig. 2(b)]. The difficulty of this method is to correctly define the basis of the nonradiating modes and their coupling constants with the radiation continuum. In the second approach, the resonator is primordially considered as an open non-Hermitian system, characterized by a complex eigenfrequency spectrum. In this approach, a small change of the resonator shape could be described as a perturbation $\delta \varepsilon(\mathbf{r})$ playing a role of the interaction potential $\hat{V}$ between modes $\left|\varphi_{a}\right\rangle$ and $\left|\varphi_{b}\right\rangle$ (see Refs. 6 and 42). In our case, a perturbation $\delta \varepsilon(\mathbf{r})$ is responsible for change in the aspect ratio of the cylindrical resonator [Fig. 2(c)]. This method is well developed for quantum mechanics and electrodynamics. ${ }^{43-47}$ It allows one to find the spectrum, eigenmodes, and interaction constants straightforwardly from the Maxwell's equations (see Appendix B). 
For the cylindrical resonator, the strong coupling between the Mie-like and Fabry-Perot-like modes is clearly manifested in the map of the SCS as avoided resonance crossing points [Figs. 1(c) and 1(d)]. The most pronounced regions of the avoided resonance crossing are marked by red ellipses in Fig. 1(d). More detailed analysis shows that in the vicinity of the avoided resonance crossing, the $Q$-factor of one of the coupled modes becomes very high, which corresponds to the appearance of a quasi-BIC. The dramatic increase in the $Q$-factor is a result of destructive interference between the modes with similar radiation patterns in the far field.

\subsection{Analysis of the Fano Parameter}

The scattering of light by high-index dielectric particles is governed by the Mie resonances of the structure. In the case of highly symmetric geometries, such as infinite rods ${ }^{48,49}$ spheres,${ }^{50}$ or core-shell particles, ${ }^{51}$ it has been shown by means of the Mie theory that the SCS represents a series of Fano resonances where each resonance can be described by the Fano formula. ${ }^{52,53}$ For other designs of resonators, e.g., finite dielectric cylinders, analytical Mie solution does not exist because the variables of the Maxwell's equations are not separable. However, description of the SCS by the Fano formula is still convenient but, in general, Fano parameters are introduced phenomenologically. The most challenging problem is the determination of exact expressions for Fano parameters in a concise and clear form. It is worth mentioning that the Fano formula for resonators with complicated geometries can be obtained but for special assumptions. ${ }^{54}$

In this paper, we derive an elegant analytical solution for the finite-size cylinder scattering problem by proving rigorously that SCS of a lossless dielectric cylinder irradiated by a plane wave represents the conventional Fano formula. By this approach, we investigate the strong coupling between Mie-like and Fabry-Perot-like modes and reveal the relationship between the peculiarities of the mode spectra, particularly, emergence of quasi-BICs, and the singularities of Fano parameters.

We assume harmonic time dependence of the incident field in the form $\mathbf{E}_{\mathrm{inc}} e^{-i \omega t}$ and determine SCS through the extinction cross section, which for the lossless case can be calculated using the optical theorem. ${ }^{55}$ The main idea of our approach is the expansion of scattered field amplitude into the sum of independent terms, where each term corresponds to an eigenmode of the cylinder. This becomes possible by applying the recently developed procedure of the resonant-state expansion that allows for rigorous characterization of eigenmode spectrum of open optical resonators. ${ }^{56}$ The cylinder eigenmodes (or resonant states) are treated as self-standing resonator excitations with a complex spectrum describing both the resonant frequencies $\omega_{0}$ and damping rates $\gamma$. Our straightforward but cumbersome calculations (see Supplementary Materials) show that the frequency dependence of the SCS could be rigorously described by the Fano formula and the Fano parameters could be expressed analytically through the material and geometrical parameters of the cavity:

$$
\sigma(\omega)=\frac{c^{2}}{\omega^{2}\left|\mathbf{E}_{\mathrm{inc}}\right|^{2}}\left[\frac{A}{1+q^{2}} \frac{(q+\Omega)^{2}}{1+\Omega^{2}}+I_{\mathrm{bg}}(\omega)\right],
$$

$A=c|\kappa|^{2} / 2 \gamma$, $q=-\cot \Delta$,

$\Delta=\arg (\kappa)$,

where $\Omega=\left(\omega-\omega_{0}\right) / \gamma$ is the relative frequency detuning, $q$ is the Fano asymmetry parameter, $A$ is the smooth amplitude of the peak, $\Delta$ is the resonant phase, and $I_{\mathrm{bg}}$ is the background contribution describing the nonresonant scattering terms. For $\omega$ in the vicinity of one of the eigenfrequencies $\omega_{0}-i \gamma$, frequency dispersion of Fano parameters can be neglected. The key parameter of the model, which determines both $q$ and $A$, is the coupling coefficient between the electric field of the resonant state $\mathbf{E}_{\mathrm{rs}}$ and of the incident field $\mathbf{E}_{\text {inc }}$ :

$\kappa=-\frac{\omega_{0}^{2}}{c^{2}} \int_{\text {cylinder }} \mathrm{d} \mathbf{r}\left(\varepsilon_{1}-\varepsilon_{2}\right) \mathbf{E}_{\mathrm{rs}}(\mathbf{r}) \cdot \mathbf{E}_{\mathrm{inc}}\left(\omega_{0}, \mathbf{r}\right)$.

The developed approach allows for investigation of the evolution of the Fano parameters of coupled modes in a parametric space (for different aspect ratios of the cylinder). As an example, we apply it to study the coupling between $\mathrm{TE}_{1,1,0}$ and $\mathrm{TM}_{1,1,1}$ modes, of which the SCS spectrum in the vicinity of the avoided resonance crossing is shown in Fig. 3(a). The calculated resonance positions, $Q$-factor, and Fano parameters $A$ and $q$ together with results of extraction of the same data by numerical fitting of the SCS to the Fano formula are shown in Figs. 3(b)-3(e).

Analysis of Fig. 3 reveals strong correlation between the evolution of Fano parameters and the quality factor of the highfrequency band. Foremost, $q$ tends to infinity exactly at $r / l=0.543$, where the quasi-BIC with high $Q$-factor emerges. Next, at $r / l=0.59$, where the SCS shows a narrow deep with a symmetric quasi-Lorentzian antiresonance and $q=0$, the peak amplitude $A$ decreases dramatically to a negligible but nonzero value. This means that mode excitation from the far field is strongly suppressed so the mode becomes almost dark. Importantly, we can claim that a quasi-BIC with high $Q$-factor and a dark mode with vanishing intensity manifest themselves at different values of the aspect ratio. This counterintuitive result shows that quasi-BIC differs substantially from a true BIC supported by unbound structures, which is always a dark mode. Although true BICs are invisible in the scattering spectrum, quasi-BICs can be tracked easily by controlling peak shapes in the SCS.

To gain deeper insight into a link between formation of quasi-BIC and peculiarities of Fano parameters in SCS spectra, we consider the radiation losses as a perturbation. This approach is natural and justified as we are working in the vicinity of quasi-BIC, where radiation losses are strongly suppressed. Remarkably, nonperturbative extension of this method is developed in Ref. 57. In general, radiation continuum represents a set of independent channels that we label by $\alpha$. For a single resonator, the independent channels can be attributed to the spherical multipoles. The details of the multipole analysis of quasi-BICs are provided in Sec. 2.3. The coupling amplitude $D_{\alpha}$ between the resonant state and the radiation continuum mode $\mathbf{E}_{\alpha}$ is given by (see Supplementary Materials)

$D_{\alpha}=-\frac{\omega_{0}^{2}}{c^{2}} \int_{\text {cylinder }} \mathrm{d} \mathbf{r}\left(\varepsilon_{1}-\varepsilon_{2}\right) \mathbf{E}_{\mathrm{rs}}(\mathbf{r}) \cdot \mathbf{E}_{\alpha}\left(\omega_{0}, \mathbf{r}\right)$. 

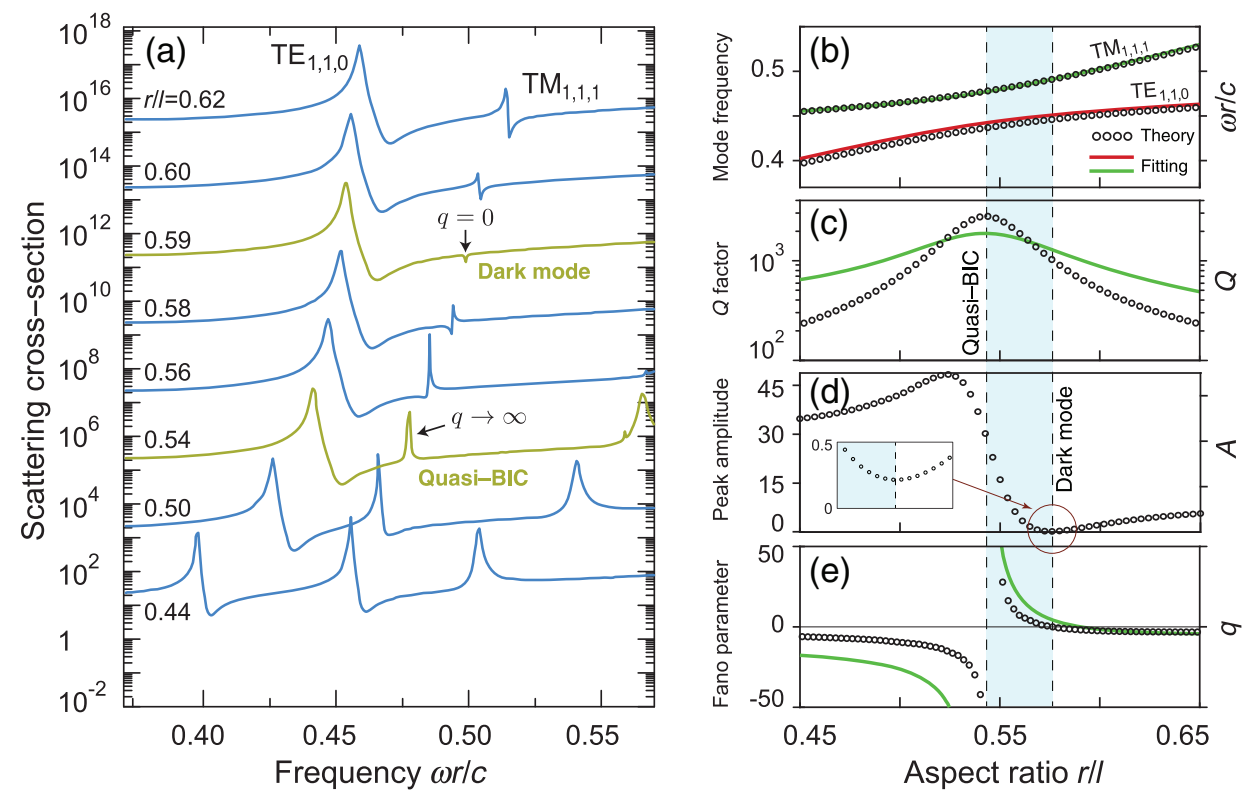

Fig. 3 Avoided resonance crossing, $Q$-factor, and Fano resonance. (a) Spectra of the normalized total SCS of the cylinder resonator as a function of its aspect ratio $r / l$ in the region of the avoided resonance crossing between the modes $\mathrm{TE}_{1,1,0}$ and $\mathrm{TM}_{1,1,1}$. (b) Peak positions for the low- and high-frequency modes in the spectra. (c) and (d) Evolution of the quality-factor $Q$, the peak amplitude $A$ [see Eq. (2)], and the Fano asymmetry parameter $q$ [see Eq. (3)] for the high-frequency mode.

According to the reciprocity theorem, the same amplitudes $D_{\alpha}$ determine coupling with the incident field. ${ }^{58}$ Following the perturbation theory, we represent each resonant state $\mathbf{E}_{\mathrm{rs}}$ as the sum of a closed resonator mode $\mathbf{E}^{(0)}$ and first-order correction $i \delta \mathbf{E}$ responsible for the radiation. Therefore, the coupling constant can be also expanded as $D_{\alpha}=D_{\alpha}^{(0)}+i \delta D_{\alpha}$. The inverse radiation lifetime $\gamma$ of the resonant state can be calculated as the sum of radiation losses into all radiation channels (like the Fermi's golden rule in quantum mechanics):

$2 \gamma=c \sum_{\alpha}\left|D_{\alpha}\right|^{2}$

Furthermore, the coupling coefficient $\kappa$ between the resonant state and the incident field [see Eq. (5)] can be decomposed into a series of independent contributions of all channels [see Supplementary Materials). For most of the eigenmodes, their radiation is mainly determined by a single dominant channel, which we denote as $D_{\text {main }}$. The remaining small amount of power goes via other channels, which we denote as $D_{\text {other }}=\sum_{\alpha}^{\prime} D_{\alpha}$ [see Fig. 4(a)]. The sum is taken over all radiation channels except the main one. In these notations, the critical behavior of the Fano asymmetry parameter $q$ is determined by the following simple conditions:

$\delta D_{\text {main }}+\delta D_{\text {other }}=0, \quad q \rightarrow \infty$,

$D_{\text {main }}^{(0)}+D_{\text {other }}^{(0)}=0, \quad q=0$.

Inspection of Eqs. (7)-(9) reveals the crucial role of the main channel in both the formation of high- $Q$ modes and critical behavior of $q$. If radiation to the main channel is completely suppressed $\left[D_{\text {main }}^{(0)}+\delta D_{\text {main }}=0\right]$, we get a quasi-BIC, characterized by minimal radiation losses. However, the Fano asymmetry parameter $q$ tends to infinity not exactly at quasi-BIC, but very close to it when the conditions of Eq. (8) are fulfilled. The dark mode $(q=0)$ is formed under the conditions of Eq. (9) when the radiation into the main and the rest channels compensates each other in the first approximation.

For deeper understanding, we focus on the particular example and investigate the evolution of coupling coefficients $D_{\alpha}$ for the high-frequency band of the avoided resonance crossing between $\mathrm{TE}_{1,1,0}$ and $\mathrm{TM}_{1,1,1}$ modes. In this case, the main channel represents electric dipole radiation and other channels are dominated by the magnetic quadrupole radiation. The comparison of the evolution of the inverse radiation lifetime $\gamma$, phase $\Delta$, and the amplitudes $D^{(0)}$ and $\delta D$ for different channels with respect to $r / l$ is shown in Figs. 4(b)-4(e), respectively.

Figures 4(d) and 4(e) show that the radiation to the main channel is suppressed by virtue of coupling between $\mathrm{TE}_{1,1,0}$ and $\mathrm{TM}_{1,1,1}$ modes, and it completely vanishes at $r / l=$ 0.543 where the quasi-BIC emerges. Importantly, in this regime $\delta D_{\text {main }}$ nulls simultaneously with $D_{\text {main }}^{(0)}$ as both of them are proportional to the rate of radiation into the main channel. Under further change in the aspect ratio $r / l$, the amplitudes $D_{\text {main }}^{(0)}$ and $\delta D_{\text {main }}$ become negative and continue to decrease. At $r / l=0.546$, the perturbation $\delta D_{\text {main }}$ compensates $\delta D_{\text {other }}$, and $q$ goes to infinity [Eq. (8)]. For further increase in the aspect ratio, the unperturbed amplitude $D_{\text {other }}^{(0)}$ of the nondominant channels becomes exactly opposite to $D_{\text {main }}^{(0)}$ at $r / l=0.575$. Thus, conditions of Eq. (9) are satisfied and $q=0$. Therefore, the quasi-BIC and dark modes did not appear at the same value of the aspect ratio because of the contribution of other nondominant channels of radiation losses. 


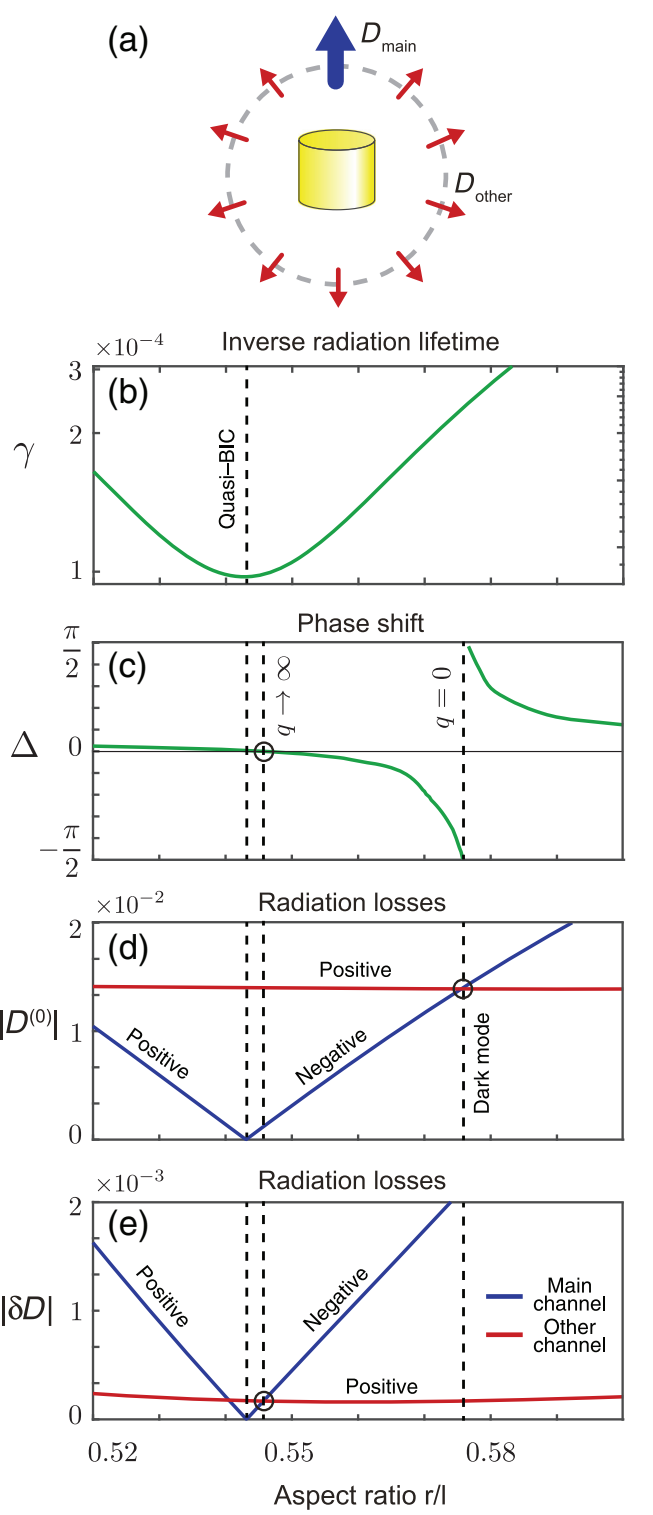

Fig. 4 Relationship between Fano parameter and $Q$-factor. (a) Artistic view of the open resonator which radiates into the main open channel $D_{\text {main }}$ and other minor channels $D_{\text {other }}$. (b) Dependence of the inverse radiation lifetime $\gamma$ on the cylinder aspect ratio for the high-frequency mode (see Fig. 3). (c) Dependence of the phase shift $\Delta$ on the aspect ratio $r / l$.

(d) Dependence of the zero-order radiation amplitudes $D_{\text {main }}^{(0)}$ and $D_{\text {other }}^{(0)}$ on the aspect ratio $r / l$. (e) Dependence of the first-order corrections to the radiation amplitudes, $\delta D_{\text {main }}$ and $\delta D_{\text {other }}$ on the aspect ratio $r / l$.

\subsection{Multipole Analysis}

To gain deeper insight into the physics of quasi-BIC in a single resonator, we illustrate cancelation of its radiation losses through the dominant channel in term of multipoles. The far field of a single resonator could be expanded into a multipole series of vector spherical harmonics. Each harmonic plays a role as an independent radiation channel. One-to-one correspondence between eigenmodes and spherical multipoles can be established only for spherical resonators. Any mode of other

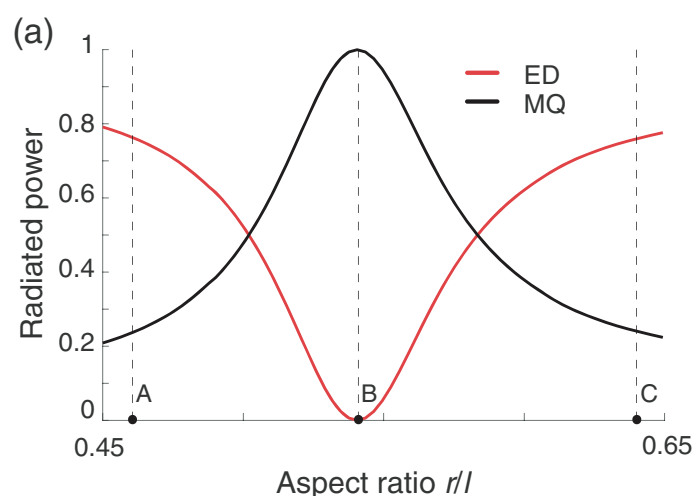

(b)
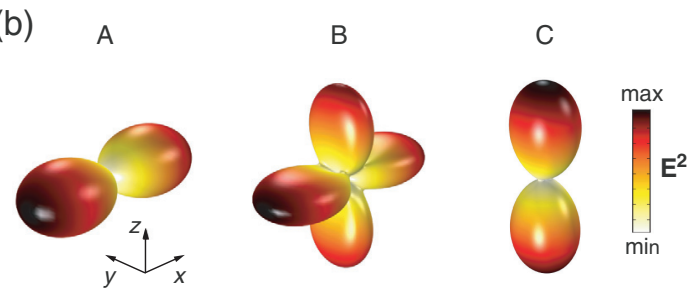

Fig. 5 Multipole decomposition for $\mathrm{TM}_{1,1,1}$ mode. (a) Contribution of the electric dipole and magnetic quadrupole to the radiated power of $\mathrm{TM}_{1,1,1}$ mode. (b) Far-field radiation patterns of $\mathrm{TM}_{1,1,1}$ mode for different aspect ratios. Panel B corresponds to the quasi-BIC.

resonators is always contributed by the infinite number of multipoles. However, in this infinite series it is possible to distinguish the dominant term (dominant channel) making the main contribution to the radiated power.

It is possible to show using the group symmetry analysis that for the mode $\mathrm{TM}_{1,1,1}$ the main contribution to the radiation energy is given by electric dipole moment and the remaining part of the energy is mainly radiated through the magnetic quadrupole moment. For the aspect ratio $r / l$ of the quasi-BIC, radiation through the dipole channel becomes negligible, the dominant radiation channel is the magnetic quadrupole [see Fig. 5(a)] and the radiation pattern changes dramatically [see Fig. 5(b)]. It is possible to show that all other quasi-BICs demonstrate similar behavior in far field. ${ }^{59}$ Therefore, quasi-BICs in single resonators are manifested not only in the scattering spectra as a singularity of the Fano asymmetry parameter but also in the far field, since the radiation pattern changes dramatically.

\subsection{Two-Band Model of Strong Mode Coupling}

In this section, we discuss the mechanism of strong coupling between modes in a cylindrical resonator and the reason for complete suppression of radiation to the main channel. As shown in the previous section, mode coupling is realized not in real but in parametric space. This means that the cylinder aspect ratio represents the parameter determining the strength of interaction between modes. Therefore, coupling between the modes is governed by the perturbation of cylinder permittivity [see Fig. 2(c)].

Generally, this perturbation mixes all resonant states of the cylinder. New resonant states of the perturbed resonator can be found by means of the resonant-state expansion (see Appendix B), which is a special rigorous technique that allows 
for careful investigation of the spectrum of open systems. However, in the vicinity of an avoided resonance crossing the interaction between modes involves only two of them. Therefore, the general form of the resonant-state expansion can be reduced to a two-band model.

We start from the two of uncoupled cylinder eigenfunctions $\left|\varphi_{\mathrm{a}}\right\rangle$ and $\left|\varphi_{\mathrm{b}}\right\rangle$ with eigenfrequencies $\omega_{\mathrm{a}}-i \gamma_{\mathrm{a}}$ and $\omega_{\mathrm{b}}-i \gamma_{\mathrm{b}}$, respectively. They undergo strong coupling and the perturbed eigenfunction with eigenfrequency $\bar{\omega}$ represents their linear combination $|\varphi\rangle=C_{\mathrm{a}}\left|\varphi_{\mathrm{a}}\right\rangle+C_{\mathrm{b}}\left|\varphi_{\mathrm{b}}\right\rangle$, where coefficients $C_{\mathrm{a}, \mathrm{b}}$ are, in general, complex. The two-band model can be written as a generalized eigenvalue problem: ${ }^{56}$

$\left[\begin{array}{cc}\omega_{\mathrm{a}}-i \gamma_{\mathrm{a}} & 0 \\ 0 & \omega_{\mathrm{b}}-i \gamma_{\mathrm{b}}\end{array}\right]\left[\begin{array}{c}C_{\mathrm{a}} \\ C_{\mathrm{b}}\end{array}\right]=\bar{\omega}\left[\begin{array}{cc}1+V_{\mathrm{aa}} & V_{\mathrm{ab}} \\ V_{\mathrm{ba}} & 1+V_{\mathrm{bb}}\end{array}\right]\left[\begin{array}{c}C_{\mathrm{a}} \\ C_{\mathrm{b}}\end{array}\right]$

Here the perturbation is determined by the symmetric matrix with complex-valued elements that make the problem non-Hermitian:

$V_{i j}=\frac{1}{2} \int \mathrm{d} \mathbf{r} \delta \varepsilon(\mathbf{r}) \mathbf{E}_{\mathrm{rs}}^{i}(\mathbf{r}) \cdot \mathbf{E}_{\mathrm{rs}}^{j}(\mathbf{r}), \quad i, j=a, b$.

Figure 6 shows perfect coincidence of the exact dispersion of two coupled modes and the two-band model results. Since both modes possess sufficiently low radiative losses, when we change cylinder aspect ratio they couple predominantly in the near-field region inside the resonator. Therefore, when the coupling is maximal, the coefficients become $C_{\mathrm{a}}=1$, $C_{\mathrm{b}}= \pm 1$ for low- and high-frequency modes in Fig. 6, respectively. We recall that $\left|\varphi_{a}\right\rangle$ and $\left|\varphi_{b}\right\rangle$ are characterized by similar far-field patterns, as they have the same mode of symmetry with respect to azimuthal direction and inversion symmetry of structure (see Appendix B). This means that far-field distribution of radiation of the high-frequency mode in the strong coupling regime is almost suppressed, i.e., $\left.\left[\left|\varphi_{b}\right\rangle-\left|\varphi_{a}\right\rangle\right]\right|_{r \rightarrow \infty} \simeq 0$. Therefore, the radiation to the main channel for this value of $r / l$ becomes completely forbidden, which explains the formation of high- $Q$ quasi-BIC mode and the dark mode discussed in the previous section.

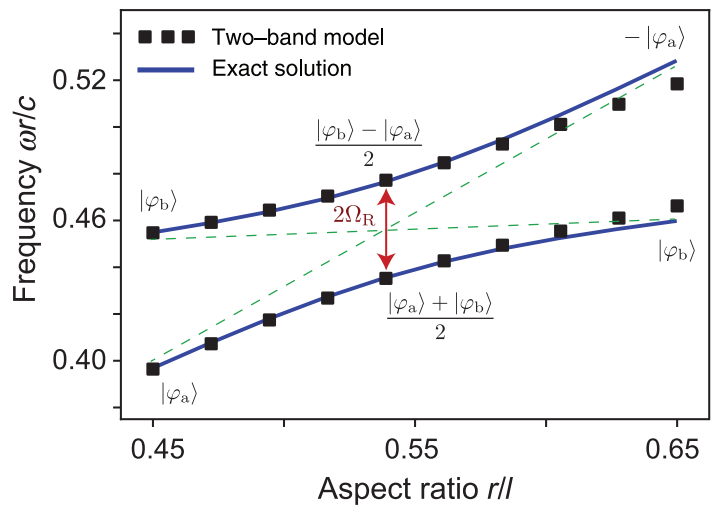

Fig. 6 Two-band approximation of strong mode coupling. Comparison of the exact solution and approximate two-band model of strong coupling between the modes $T E_{1,1,0}$ and $T M_{1,1,1}$. Green dashed lines are visual guides.
The conventional method to characterize strength of mode coupling is the Rabi frequency $\Omega_{R}$, which is a half of minimal distance between the dispersion curves of coupled modes (see Fig. 6). For the two resonances to be spectrally separable, the minimum mode-splitting needs to be greater than the sum of the half linewidths of the modes, which is a necessary condition to observe strong coupling. ${ }^{60}$ For the avoided resonance crossing under consideration $\Omega_{\mathrm{R}} r / c=0.018$, which is 35 times higher than the sum of the half linewidths, and which clearly manifests the strong coupling of modes.

\subsection{Effect of Material Losses}

For the cylindrical resonator analyzed above, we neglect the material losses and take into account only the radiative ones. Here, we analyze the effect of material losses on the quality factor of quasi-BIC and on the conditions of strong coupling. Figure 7(a) shows the dependence of the total quality factor $Q$ on the aspect ratio for the high-frequency mode in the vicinity of the avoided crossing regime between the modes $\mathrm{TE}_{1,1,0}$ and $\mathrm{TM}_{1,1,1}$ at different material loss level. One can see that the $Q$ factor strongly depends on the material losses and could be substantially decreased. In the presence of material losses, the total $Q_{\text {tot }}$ factor could be estimated as

$Q_{\text {tot }}^{-1}=Q_{\text {rad }}^{-1}+Q_{\text {mat }}^{-1}$,
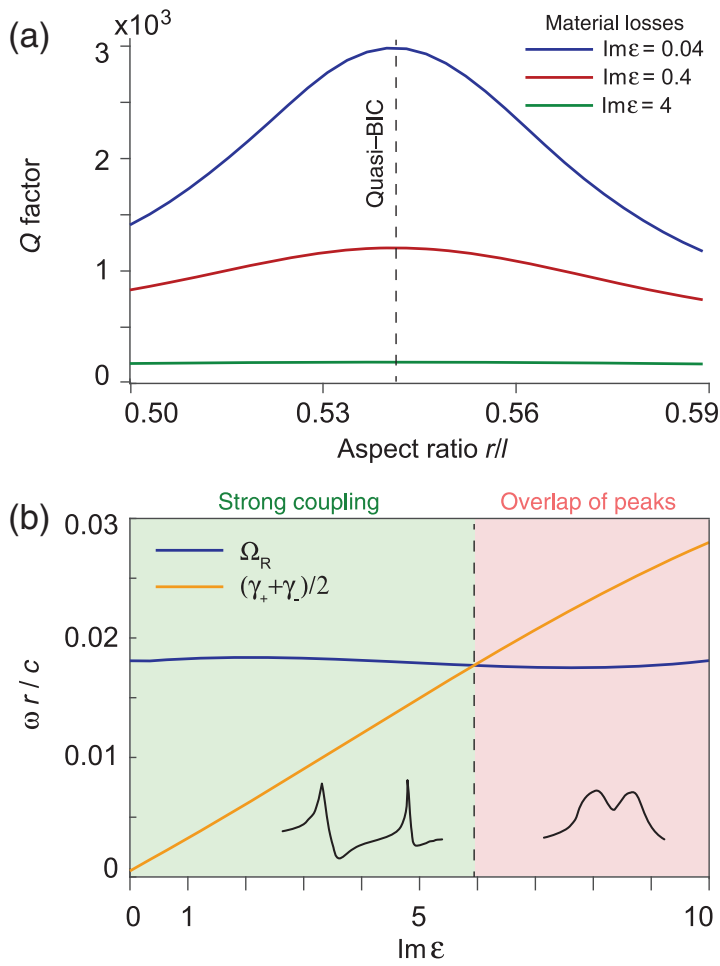

Fig. 7 Effect of material losses on the regime of strong coupling and quasi-BIC. (a) Dependence of the total quality-factor $Q$ on the aspect ratio for various levels of material losses. (b) Dependence of Rabi frequency and sum of half linewidths of the coupled modes on the level of material losses. Insets visualize the ratio between $\Omega_{\mathrm{R}}$ and linewidths. Here, $\gamma_{+}$and $\gamma_{-}$are the damping rates of the modes of the diagonalized Hamiltonian [Eq. (10)]. 
where $Q_{\text {rad }}$ and $Q_{\text {mat }}$ are responsible for the radiative and material losses, respectively. Therefore, the results obtained for the lossless cavity are justified and $Q_{\text {tot }} \approx Q_{\text {rad }}$ if the radiative losses are dominant $\left(Q_{\text {rad }}>Q_{\text {mat }}\right)$.

Since material losses decrease the $Q$-factor, they affect the strength of mode coupling as well. Figure 7(b) shows how the Rabi frequency $\Omega_{\mathrm{R}}$ and the sum of the half linewidths change depending on the level of material losses. The strong coupling regime breaks when the resonance becomes spectrally inseparable, which is realized for $\operatorname{Im} \varepsilon=6$. Therefore, even for relative high absorption the strong coupling can be realized, which is extremely useful for the experimental measurements described in the next section.

\subsection{Experimental Results}

Finally, we perform the experimental study to demonstrate the existence of the avoided crossing regime between the $\mathrm{TE}_{1,1,0}$ and $\mathrm{TM}_{1,1,1}$ resonances in the microwave frequency range. In the experiment, the plastic cylindrical vessel filled with water is placed in the middle between two antennas. The aspect ratio of the cylindrical resonator is defined by the amount of water. A photo of the experimental setup is shown in Fig. 8(a) (see Appendix A for details). The resonator is excited by TE polarized electromagnetic wave incident perpendicular to the cylinder axis $z$ [see Fig. 8(a)]. The measured dependence of the SCS of the cylindrical resonator on its aspect ratio is shown in Fig. 8(b). The results of the numerical simulations taking into account the losses in water are shown in Fig. 8(c). One can see that the experimental positions of the resonances are in a good agreement with the real part of eigenfrequencies (marked by white circles) calculated using the resonant-state expansion method (see Appendix B for details). In spite of losses in water, which broaden the resonances, the avoided crossing regime between the $\mathrm{TE}_{1,1,0}$ and $\mathrm{TM}_{1,1,1}$ modes and suppression of SCS clearly manifest themselves for the aspect ratio in the range of $0.5<r / l<0.6$. Discrepancies between the measured and calculated maps of SCS could be explained by an imperfect plane wave radiated by a horn antenna and parasitic scattering from the auxiliary equipment (holder of the resonator and plastic cylindrical vessel).

\section{Discussion}

As we mentioned above, a true BIC is mathematical abstraction and it is not practically implementable. However for periodic photonic structures with large numbers of periods, the radiation of high- $Q$ states could be almost suppressed, being much less than other loss mechanisms in the system. Such states are closest to the true BICs. Here, we demonstrate that the radiation losses can be substantially suppressed, being much smaller than other losses, via a BIC-inspired mechanism even in a single isolated resonator. Therefore, BIC in a finite size periodic structure and quasi-BIC in a single resonator could be indistinguishable in practice if their radiation losses will be strongly suppressed. We believe that the proposed concept of quasi-BIC in a single resonator is more favorable for compact nanophotonic applications and more easily implementable than other designs.

The developed theory predicts that the shape and amplitude of the SCS spectra represent an unambiguous indicator of the quasi-BIC. Namely, both regimes of $q=0$ and $q \rightarrow \infty$ describe important features inherent to a true BIC. The latter condition $q \rightarrow \infty$ practically coincides with the emergence of a quasiBIC. Therefore, a quasi-BIC could be recognized in the experimental spectra by the naked eye since the asymmetry of the Fano resonance is easily distinguishable without fitting.

The difference in the aspect ratios corresponding to quasiBIC and singularity of $q$ is determined by the magnitude of radiation losses to nondominant channels. The difference becomes negligible with an increase in the permittivity of the resonator. Even for high-index dielectric nanostructures in the visible and near-IR ranges, i.e., $\varepsilon \sim 10$ to 12 , the relative difference between aspect ratios corresponding to the singular $q$ and the maximal $Q$-factor is $<5 \%$. The predicted strong coupling regime and peculiarities of the Fano parameters could be observed in a wide spectral range from the visible to centimeter wavelengths. These results lift the veil on the nature of quasi-BICs in single resonators and emphasize the profound (a)

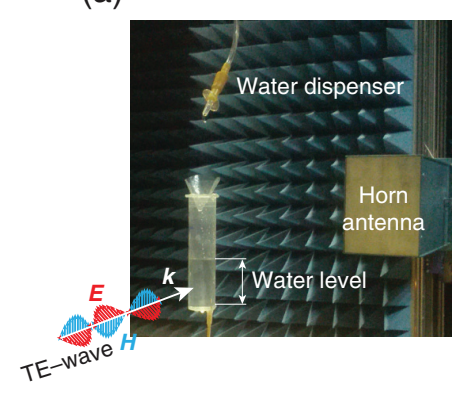

(b)

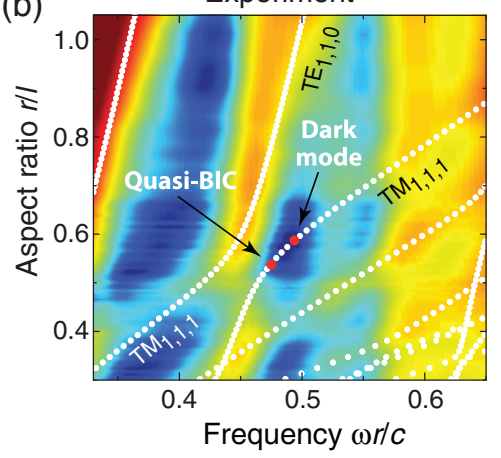

(c)

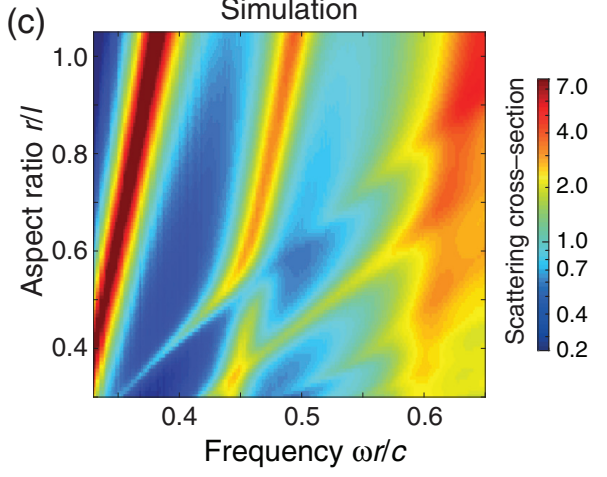

Fig. 8 Experimental results. (a) Experimental setup for the measurement of SCS spectra of the cylindrical resonator filled with water depending on its aspect ratio $r / /$ and frequency $\omega r / c$. (b) Measured SCS map demonstrating the avoided crossing regime between $\mathrm{TE}_{1,1,0}$ and $\mathrm{TM}_{1,1,1}$ resonances. The circles are the real part of eigenfrequencies obtained from the resonant-state expansion method for a dielectric cylinder with the permittivity $\varepsilon_{1}=80$ embedded in air $\left(\varepsilon_{2}=1\right)$. (c) Calculated SCS map of the cylindrical resonator filled with water depending on the frequency $\omega r / c$ and aspect ratio $r / I$. The frequency dispersion of the water permittivity is taken from Ref. 61. 
relationship between the shape of Fano peak and emergence of bound states in the continuum.

Remarkably, for BICs in periodic photonic structures, the nondominant channels are absent because the radiation continuum is discretized. ${ }^{32}$ Thus, the leaky modes interact with only one channel, which in the simplest case represents the zero-order diffraction. ${ }^{62}$ Therefore, leaky states can transform to true BICs, when the radiation to the main channel is completely forbidden. Since for true BIC, the system of Eqs. (8) and (9) is satisfied simultaneously, the Fano parameter $q$ becomes illdefined, which corresponds to the collapse of Fano resonance. Furthermore, true BIC is always a dark mode, which can be easily understood from energy consumption arguments-BIC does not radiate at all, thus we are not able to pump it. But for finite-size dielectric cylinders, the collapse of Fano resonance is not manifested; instead of this, Fano parameter monotonically evolves as the cylinder aspect ratio changes and consistently passes the values $q= \pm \infty$ and $q=0$, where the mode exhibits features of a true BIC state.

Recently, the study of resonant dielectric nanostructures has been established as a research direction in modern nanoscale optics and metamaterial-inspired nanophotonics due to their optically induced electric and magnetic Mie resonances. ${ }^{63,64}$ However, the $Q$-factor of Mie resonances is about ten times farther from the values achieved in WGM resonators, photonic crystals, or Bragg cavities. The proposed mechanism of strong mode coupling in single high-index dielectric resonators accompanied by the emergence of quasi-BIC helps to substantially extend functionality of all-dielectric nanophotonics. This opens new horizons for active and passive nanoscale metadevices including low-threshold nanolasers, biosensors, on-chip parametric amplifiers, and nanophotonics quantum circuits.

\section{Conclusion}

We have demonstrated that a subwavelength homogeneous dielectric resonator can support strongly interacting modes. We have shown that the strong coupling regime is accompanied by the formation of a quasi-BIC when the radiative losses are almost suppressed due to the Friedrich-Wintgen destructive interference. The analysis of the SCS reveals an abrupt change in the Fano asymmetry parameter from minus to plus infinity in the vicinity of quasi-BICs. Therefore, the quasi-BIC manifests itself in scattering spectra by the symmetric Lorentzian shape. Appearance of quasi-BIC is accompanied by drastic change of far-field radiation pattern explained by suppression of the radiation through the dominant multipole. This singularity could be used as an indication of quasi-BIC. In contrast to true BIC, the Fano resonance feature for quasi-BIC does not vanish completely, since it is not completely decoupled from the radiation continuum. We have confirmed our theoretical results in a microwave experiment using a cylindrical resonator filled with water. Our results open new horizons for active and passive optical nanodevices including efficient biosensors, low threshold nanolasers, perfect filters, waveguides, and nanoantennas.

\section{Appendix A: Experimental Approach}

The sample used for the experimental study of SCS is a hollow plastic cylinder opened from the top with the radius of $r=20.25 \mathrm{~mm}$ and height of $l=160 \mathrm{~mm}$. The thickness of the cylinder wall is $w=1.5 \mathrm{~mm}$. As a dielectric material to fill the cylinder, we employed a distilled water that is characterized by permittivity of $\varepsilon_{1} \approx 80$ at room temperature. ${ }^{61}$ Forward scattering measurements were performed in an anechoic chamber. ${ }^{65}$ A pair of wideband horn antennas (TRIM 0.75 to $18 \mathrm{GHz}$; DR) were positioned facing each other at a distance of $4 \mathrm{~m}$ with the sample placed at the midpoint; see Fig. 8(a). The measurement used a two ports vector network analyzer (VNA; Agilent E8362C) transmitting a continuous wave. The first antenna was connected to the first port of the VNA and provided a near plane-wave excitation in the frequency range of 0.8 to $5 \mathrm{GHz}$. The second horn antenna connected to the second port of the VNA was employed as a receiver. The frequency range of 0.8 to $5 \mathrm{GHz}$ was swept using 10,001 frequency points. Eight such sweeps were averaged for each of the sample measurement, background measurement, and calibration measurement. Calibration measurements were performed using a metal sphere with the radius of $7.5 \mathrm{~mm}$. During the sample measurements, we added the water to the cylinder changing its aspect ratio $r / l$ from 0.125 to 2.5 with the average step of 0.01 . The optical theorem was used to calculate the SCS from the imaginary part of the measured forward scattering amplitude. ${ }^{66}$ To suppress the effects of multiple reflections between the sample and the antennas, the postprocessing of measured data by means of time-domain gating was employed. ${ }^{67}$

\section{Appendix B: Analytical Model}

We calculate the spectrum of complex eigenfrequencies of a dielectric cylindrical resonator by applying the rigorous perturbative method, the resonant-state expansion. ${ }^{44}$ We expand the fields $\mathbf{E}_{j}(j=n, k, p)$ of eigenmodes of the cylindrical resonator over the eigenfunctions $\mathbf{E}_{\alpha}^{(0)}$ of a homogeneous dielectric sphere with the same value of permittivity as for the cylindrical resonator,

$\mathbf{E}_{j}=\sum_{\alpha} b_{\alpha}^{j} \mathbf{E}_{\alpha}^{(0)}$,

where $\mathbf{E}_{\alpha}^{(0)}$ satisfies the Maxwell's equations with boundary conditions of outgoing waves:

$\nabla \times \nabla \times \mathbf{E}_{\alpha}^{(0)}=\varepsilon(\mathbf{r}) \frac{\omega_{\alpha}^{2}}{c^{2}} \mathbf{E}_{\alpha}^{(0)}$.

Resonant states $\mathbf{E}_{j}$ satisfy the perturbed equation

$\nabla \times \nabla \times \mathbf{E}_{j}=[\varepsilon(\mathbf{r})+\delta \varepsilon(\mathbf{r})] \frac{\Omega_{j}^{2}}{c^{2}} \mathbf{E}_{j}$,

where $\delta \varepsilon(\mathbf{r})$ is a perturbation that transforms a sphere into an inscribed cylinder.

The problem is non-Hermitian because of outgoing boundary conditions. Therefore, the eigenvectors grow exponentially at large distances, and their proper normalization deviates from the standard Hermitian anzatz. ${ }^{44}$ However, $\mathbf{E}_{\alpha}^{(0)}$ forms a complete set inside the region of a dielectric sphere and we use them as a basis.

The problem is reduced to the matrix equation ${ }^{44}$

$$
\frac{1}{\omega_{\alpha}} \sum_{\beta}\left(\delta_{\alpha \beta}+V_{\alpha \beta}\right) b_{\beta}^{j}=\frac{1}{\Omega_{j}} b_{\alpha}^{j},
$$


with the elements of the perturbation matrix $V_{\alpha \beta}$ as defined in Eq. (11). We should note that here the operator $\hat{V}$ is responsible for the transformation of the sphere into the cylinder. Thus, this operator $\hat{V}$ differs from those defined in Figs. 2(b) and 2(c).

The resonant-state expansion represents a generalization of the Brillouin-Wigner perturbation theory for non-Hermitian systems. ${ }^{46}$ Therefore, numerical accuracy is determined by the size of the basis set $N$. We choose the basis in such a way that for a given orbital number $l$, azimuthal number $n$, and parity, we select all resonant states with frequencies lying inside the circle $|\omega R / c|<10$, where $R$ is the radius of the sphere that describes the cylinder. We consider $l<80$, which results in $N=1035$ that is enough to achieve $99.9 \%$ accuracy for calculation of real part of frequencies. Since the perturbation $V_{\alpha \beta}$ conserves the axial symmetry and mirror $(z \rightarrow-z)$ symmetry, we study problem for each azimuthal index $n$ and each parity independently.

The dependence of the complex spectrum of eigenmodes with azimuthal indices $n=0, \pm 1$, which are even with respect to up-down reflection symmetry, versus the cylinder aspect ratio $r / l$, is shown in Fig. 9 by dotted lines. Dot sizes are proportional to the $Q$-factor. We can clearly observe multiple avoided resonance crossings between modes with the same azimuthal number. In the vicinity of an avoided crossing point, the $Q$-factor of one of the coupled modes dramatically increases, which confirms the results of SCS calculations [see Figs. 1(c) and 3(c)].

In the high-frequency region, the behavior of interaction between modes becomes more complicated, e.g., $Q$-factors of some of $n= \pm 1$ modes remain high in a broad range of parameters $x$ and $r / l$, as shown in Fig. 9. We explain this phenomenon as strong coupling between three and more eigenmodes with complex spectrum. This broadband high- $Q$ regime will be the subject of our further investigations. In this article, we restrict our studies to the mechanism of strong coupling between two eigenmodes, because it illustrates the basic peculiarities of spectrum of subwavelength dielectric resonators.

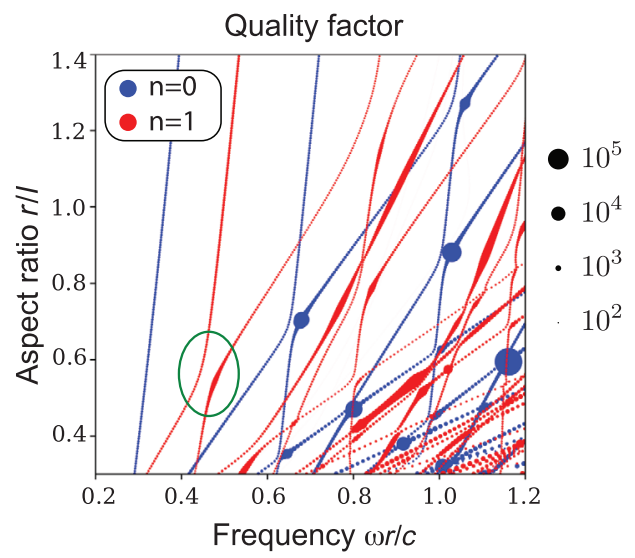

Fig. 9 Complex spectrum of eigenmodes. The spectrum is shown for the modes with the azimuthal index $n=0$ (red dotted lines) and $n= \pm 1$ (blue dotted lines), which are even with respect to $(z \rightarrow-z)$ symmetry. Dot sizes are proportional to $Q$-factor. The region of the avoided crossing between modes $T E_{1,1,0}$ and $\mathrm{TM}_{1,1,1}$ is marked by the green ellipse. Calculations are performed by using the resonant-state expansion method.

\section{Acknowledgments}

We acknowledge fruitful discussions with H. Atwater, I.V. Shadrivov, P.A. Belov, A.N. Poddubny, A. Polman, and A. Moroz. The numerical calculations were performed with support from the Ministry of Education and Science of the Russian Federation (Project 3.1500.2017/4.6) and the Australian Research Council. The experimental study of the cylinder SCS in the microwave frequency range was supported by the Russian Science Foundation (17-79-20379). The analytical calculations with resonant-state expansion method were performed with support from the Russian Science Foundation (17-12-01581). A. A. B., K. L. K. and Z. F. S. acknowledge support from the Foundation for the Advancement of Theoretical Physics and Mathematics "BASIS" (Russia).

\section{References}

1. K. J. Vahala, "Optical microcavities," Nature 424, 839-846 (2003).

2. B. Min et al., "High- $Q$ surface-plasmon-polariton whisperinggallery microcavity," Nature 457, 455-458 (2009).

3. S.-H. Kwon et al., "Subwavelength plasmonic lasing from a semiconductor nanodisk with silver nanopan cavity," Nano Lett. 10(9), 3679-3683 (2010).

4. Y. Akahane et al., "High- $Q$ photonic nanocavity in a twodimensional photonic crystal," Nature 425, 944-947 (2003).

5. A. Matsko and V. Ilchenko, "Optical resonators with whisperinggallery modes-part I: basics," IEEE J. Sel. Top. Quantum Electron. 12(1), 3-14 (2006).

6. H. M. Lai et al., "Effect of perturbations on the widths of narrow morphology-dependent resonances in Mie scattering," J. Opt. Soc. Am. B 8(9), 1962-1973 (1991).

7. S. G. Johnson et al., "Multipole-cancellation mechanism for highQ cavities in the absence of a complete photonic band gap," Appl. Phys. Lett. 78(22), 3388-3390 (2001).

8. M. V. Rybin et al., "High-q supercavity modes in subwavelength dielectric resonators," Phys. Rev. Lett. 119(24), 243901 (2017).

9. J. Von Neuman and E. Wigner, "Uber merkwurdige diskrete Eigenwerte. Uber das Verhalten von Eigenwerten bei adiabatischen Prozessen," Phys. Z. 30, 467-470 (1929).

10. H. Friedrich and D. Wintgen, "Interfering resonances and bound states in the continuum," Phys. Rev. A 32(6), 3231-3242 (1985).

11. R. Parker, "Resonance effects in wake shedding from parallel plates: some experimental observations," J. Sound Vib. 4(1), 62-72 (1966).

12. R. Parker, "Resonance effects in wake shedding from parallel plates: calculation of resonant frequencies," J. Sound Vib. 5(2), 330-343 (1967).

13. A. A. Lyapina et al., "Bound states in the continuum in open acoustic resonators," J. Fluid Mech. 780, 370-387 (2015).

14. D. C. Marinica, A. G. Borisov, and S. V. Shabanov, "Bound states in the continuum in photonics," Phys. Rev. Lett. 100(18), 183902 (2008).

15. E. N. Bulgakov and A. F. Sadreev, "Bound states in the continuum in photonic waveguides inspired by defects," Phys. Rev. B 78(7), 075105 (2008).

16. R. F. Ndangali and S. V. Shabanov, "Electromagnetic bound states in the radiation continuum for periodic double arrays of subwavelength dielectric cylinders," J. Math. Phys. 51(10), 102901 (2010).

17. C. W. Hsu et al., "Observation of trapped light within the radiation continuum," Nature 499, 188-191 (2013).

18. F. Monticone and A. Alù, "Embedded photonic eigenvalues in 3D nanostructures," Phys. Rev. Lett. 112(21), 213903 (2014).

19. M. Rybin and Y. Kivshar, "Supercavity lasing," Nature 541, 164 165 (2017). 
20. Y. Plotnik et al., "Experimental observation of optical bound states in the continuum," Phys. Rev. Lett. 107(18), 183901 (2011).

21. M. I. Molina, A. E. Miroshnichenko, and Y. S. Kivshar, "Surface bound states in the continuum," Phys. Rev. Lett. 108(7), 070401 (2012).

22. G. Corrielli et al., "Observation of surface states with algebraic localization," Phys. Rev. Lett. 111(22), 220403 (2013).

23. J. Wiersig, "Formation of long-lived, scarlike modes near avoided resonance crossings in optical microcavities," Phys. Rev. Lett. 97(25), 253901 (2006).

24. J. Unterhinninghofen, J. Wiersig, and M. Hentschel, "GoosHänchen shift and localization of optical modes in deformed microcavities," Phys. Rev. E 78(1), 016201 (2008).

25. T. Lepetit and B. Kanté, "Controlling multipolar radiation with symmetries for electromagnetic bound states in the continuum," Phys. Rev. B 90(24), 241103 (2014).

26. T. Lepetit et al., "Resonance continuum coupling in high-permittivity dielectric metamaterials," Phys. Rev. B 82(19), 195307 (2010).

27. S. I. Azzam et al., "Formation of bound states in the continuum in hybrid plasmonic-photonic systems," Phys. Rev. Lett. 121(25), 253901 (2018).

28. L. Fonda, "Bound states embedded in the continuum and the formal theory of scattering," Ann. Phys. 22(1), 123-132 (1963).

29. C. S. Kim et al., "Resonant tunneling in a quantum waveguide: effect of a finite-size attractive impurity," Phys. Rev. B 60(15), 10962 (1999).

30. Z. F. Sadrieva et al., "Transition from optical bound states in the continuum to leaky resonances: role of substrate and roughness," ACS Photon. 4(4), 723-727 (2017).

31. C. Blanchard, J.-P. Hugonin, and C. Sauvan, "Fano resonances in photonic crystal slabs near optical bound states in the continuum," Phys. Rev. B 94(15), 155303 (2016).

32. E. N. Bulgakov and A. F. Sadreev, "Propagating Bloch bound states with orbital angular momentum above the light line in the array of dielectric spheres," J. Opt. Soc. Am. A 34(6), 949952 (2017).

33. I. Liberal and N. Engheta, "Near-zero refractive index photonics," Nat. Photonics 11(3), 149-158 (2017).

34. M. I. Mishchenko, "Light scattering by size-shape distributions of randomly oriented axially symmetric particles of a size comparable to a wavelength," Appl. Opt. 32(24), 4652-4666 (1993).

35. M. I. Mishchenko and L. D. Travis, "T-matrix computations of light scattering by large spheroidal particles," Opt. Commun. 109(1-2), 16-21 (1994).

36. K. Zhang and D. Li, Electromagnetic Theory for Microwaves and Optoelectronics, Springer, Berlin (2008).

37. D. J. Jackson, Classical Electrodynamics, John Wiley and Sons, New York (1998).

38. A. N. Oraevsky, "Whispering-gallery waves," Quantum Electron. 32(5), 377-400 (2002).

39. V. S. Ilchenko and A. B. Matsko, "Optical resonators with whispering-gallery modes-part II: applications," IEEE J. Sel. Top. Quantum Electron. 12(1), 15-32 (2006).

40. L. D. Landau and E. M. Lifshitz, Quantum Mechanics: NonRelativistic Theory, 3rd ed., Pergamon, Oxford (1989).

41. M. Scully and M. Zubairy, Quantum Optics, Cambridge University Press, Cambridge, United Kingdom (1997).

42. H. M. Lai et al., "Time-independent perturbation for leaking electromagnetic modes in open systems with application to resonances in microdroplets," Phys. Rev. A 41(9), 5187 (1990).

43. E. S. C. Ching et al., "Quasinormal-mode expansion for waves in open systems," Rev. Mod. Phys. 70(4), 1545-1554 (1998).

44. E. A. Muljarov, W. Langbein, and R. Zimmermann, "BrillouinWigner perturbation theory in open electromagnetic systems," EPL Europhys. Lett. 92(5), 50010 (2011).

45. Y. B. Zeldovich, "On the theory of unstable states," J. Exp. Theor. Phys. 12(3), 542-545 (1961).
46. L. Brillouin, "Les problèmes de perturbations et les champs selfconsistents," J. Phys. Radium 3(9), 373-389 (1932).

47. R. M. More, "Theory of decaying states," Phys. Rev. A 4(5), 1782 1790 (1971)

48. M. V. Rybin et al., "Mie scattering as a cascade of Fano resonances," Opt. Express 21(24), 30107-30113 (2013).

49. M. V. Rybin et al., "Switching from visibility to invisibility via Fano resonances: theory and experiment," Sci. Rep. 5, 8774 (2015).

50. M. I. Tribelsky and A. E. Miroshnichenko, "Giant in-particle field concentration and Fano resonances at light scattering by highrefractive-index particles," Phys. Rev. A 93(5), 053837 (2016).

51. X. Kong and G. Xiao, "Fano resonances in core-shell particles with high permittivity covers," in Prog. Electromagn. Res. Symp., IEEE, pp. 1715-1719 (2016).

52. U. Fano, "Effects of configuration interaction on intensities and phase shifts," Phys. Rev. 124(6), 1866-1878 (1961).

53. M. F. Limonov et al., "Fano resonances in photonics," Nat. Photonics 11(9), 543-554 (2017).

54. B. Gallinet and O. J. F. Martin, "Ab initio theory of Fano resonances in plasmonic nanostructures and metamaterials," Phys. Rev. B 83(23), 235427 (2011).

55. A. B. Evlyukhin et al., "Optical theorem and multipole scattering of light by arbitrarily shaped nanoparticles," Phys. Rev. B 94(20), 205434 (2016).

56. M. Doost, W. Langbein, and E. A. Muljarov, "Resonant-state expansion applied to three-dimensional open optical systems," Phys. Rev. A 90 (1), 013834 (2014).

57. J. S. T. Gongora, G. Favraud, and A. Fratalocchi, "Fundamental and high-order anapoles in all-dielectric metamaterials via FanoFeshbach modes competition," Nanotechnology 28(10), 104001 (2017).

58. W. Suh, Z. Wang, and S. Fan, "Temporal coupled-mode theory and the presence of non-orthogonal modes in lossless multimode cavities," IEEE J. Quantum Electron. 40(10), 1511-1518 (2004).

59. S. Muhlig et al., "Multipole analysis of eta-atoms," Metamaterials 5, 64-73 (2011)

60. L. Zhang et al., "Photonic-crystal exciton-polaritons in monolayer semiconductors," Nat. Commun. 9, 713 (2018).

61. U. Kaatze, "Complex permittivity of water as a function of frequency and temperature," J. Chem. Eng. Data 34(4), 371-374 (1989).

62. B. Zhen et al., "Topological nature of optical bound states in the continuum," Phys. Rev. Lett. 113(25), 257401 (2014).

63. A. I. Kuznetsov et al., "Optically resonant dielectric nanostructures," Science 354(6314), aag2472 (2016).

64. D. G. Baranov et al., "All-dielectric nanophotonics: the quest for better materials and fabrication techniques," Optica 4(7), 814-825 (2017).

65. G. M. Larsson, "Wideband measurements of the forward RCS and the extinction cross section," ACES J. 28(12), 1145-1152 (2013).

66. C. F. Bohren and D. R. Huffman, Absorption and Scattering of Light by Small Particles, John Wiley and Sons, New York (2008).

67. C. Larsson et al., "Extinction cross section measurements," in Proc. of Nordic Conf. on Radio Science and Communications, pp. 127-129 (2008).

Andrey A. Bogdanov received his $\mathrm{PhD}$ in 2012 from the loffe Institute, Russia, in the field of quantum cascade lasers. He is an assistant professor at the ITMO University, Russia. His current research efforts focus on theoretical investigation of optical nanoresonators and microcavities, bound states in the continuum, and surface waves in metasurfaces.

Kirill L. Koshelev is a PhD candidate at the Nonlinear Physics Centre, Australian National University. He received his MS degree in 2017 from 
the Academic University, Russia. His major research interest is in the field of theoretical analysis and designing of photonic nanostructures, nonlinear frequency generation, and bound states in the continuum.

Yuri S. Kivshar is a full-time professor and the head of the Nonlinear Physics Centre, Australian National University. He is a fellow of AA, AIP, OSA, APS, InstP. He is a world leader in nonlinear physics, nonlinear photonics, and physics of metamaterials. In the last few years, his research has focused on metaoptics and all-dielectric nanophotonics with special interest in nonlinear, active, and topological phenomena at the nanoscale.

Biographies of the other authors are not available. 